\title{
A tripled fixed point theorem for semigroups of Lipschitzian mappings on metric spaces with uniform normal structure
}

\section{Ahmed H Soliman*}

\section{"Correspondence:}

ahsolimanm@gmail.com

Present address: Department of Mathematics, Faculty of Science,

King Khaled University, Abha, 9004, Saudi Arabia

Department of Mathematics, Faculty of Science, Al-Azhar University, Assiut, 71511, Egypt

\section{空 Springer}

\begin{abstract}
In this work, we establish a tripled fixed point theorem for an asymptotically regular one-parameter semigroup $\mathfrak{\Im}=\{F(t): t \in G$, where $G$ is an unbounded subset of $[0, \infty)\}$ of Lipschitzian self-mappings on $X \times X \times X$ in a complete bounded metric space $X$ with uniform normal structure.
\end{abstract}

Keywords: coupled fixed point; tripled fixed point; asymptotically regular semigroup; uniform normal structure; convexity structure

\section{Introduction}

The classical Banach contraction principle proved in complete metric spaces continues to be an indispensable and effective tool in theory as well as applications, which guarantees the existence and uniqueness of fixed points of contraction self-mappings besides offering a constructive procedure to compute the fixed point of the underlying mapping. There already exists an extensive literature on this topic. Keeping in view the relevance of this paper, we refer to [1-5].

In 2006, Bhaskar and Lakshmikantham [6] initiated the idea of a coupled fixed point in partially ordered metric spaces and proved some interesting coupled fixed point theorems for a mapping satisfying the mixed monotone property. Many authors obtained important coupled fixed point (see [6-9]). In this continuation, Lakshmikantham and Ciric [8] introduced coupled common fixed point theorems for nonlinear $\phi$-contraction mappings in partially ordered complete metric spaces which indeed generalize the corresponding fixed point theorems contained in Bhaskar and Lakshmikantham [6]. Recently, Samet and Vetro [10] introduced the concept of fixed point of $\mathrm{N}$-order for nonlinear mappings in complete metric spaces. They obtained the existence and uniqueness theorems for contractive type mappings. Their results generalized and extended coupled fixed point theorems established by Bhaskar and Lakshmikantham [6].

On the other hand, in 1989, Khamsi [11] defined normal and uniform normal structure for metric spaces and proved that if $(X, d)$ is a complete bounded metric space with uniform normal structure, then it has the fixed point property for nonexpansive mappings and a kind of intersection property which extends the result of Maluta [12] to metric spaces. In 1995, Lim and Hong-Kun Xu [13] proved a fixed point theorem for uniformly Lipschitzian mappings in metric spaces with both property $(P)$ and uniform normal structure, which extends the result of Khamsi [11]. This is the metric space version of Casini

(O2013 Soliman; licensee Springer. This is an Open Access article distributed under the terms of the Creative Commons Attribution License (http://creativecommons.org/licenses/by/2.0), which permits unrestricted use, distribution, and reproduction in any medium, provided the original work is properly cited. 
and Maluta's theorem [2]. In 2007, Jen-Chih Yao and Lu-Chuan Zeng [14] established a fixed point theorem for an asymptotically regular semigroup of uniformly Lipschitzian mappings with property $(*)$ in a complete bounded metric space with uniform normal structure which extends the results of Lim and Hong-Kun Xu [13]. Recently, Imdad and Soliman [15] introduced fixed point theorems for an asymptotically regular semigroup of uniformly generalized Lipschitzian mappings which generalize the results due to Jen-Chih Yao and Lu-Chuan Zeng [14].

In the present paper, we prove that asymptotically regular one parameter semigroups $\Im=\{F(t): t \in G$, where $G$, an unbounded subset of $[0, \infty)\}$ of Lipschitzian self-mappings on $X \times X \times X$, has a tripled fixed point, where $X$ denotes a complete bounded metric space with uniform normal structure. Also, some corollaries of our results are presented.

\section{Preliminaries}

Definition 2.1 [6] An element $(x, y) \in X \times X$ is called a coupled fixed point of the mapping $F: X \times X \rightarrow X$ if

$$
F(x, y)=x \quad \text { and } \quad F(y, x)=y .
$$

Theorem 2.1 [6] Let $(X, \leq)$ be a partially ordered set and suppose there is a metric $d$ on $X$ such that $(X, d)$ is a complete metric space. Let $F: X \times X \rightarrow X$ be a continuous mapping having the mixed monotone property on $X$. Assume that there exists a constant $k \in[0,1)$ with

$$
d(F(x, y), F(u, v)) \leq \frac{k}{2}[d(x, u)+d(y, v)] \quad \forall x \geq u, y \leq v .
$$

If there exist $x_{0}, y_{0} \in X$ such that $x_{0} \leq F\left(x_{0}, y_{0}\right)$ and $y_{0} \geq F\left(y_{0}, x_{0}\right)$, then there exist $x, y \in X$ such that $x=F(x, y)$ and $y=F(y, x)$.

Definition 2.2 [10] An element $(x, y, z) \in X \times X \times X$ is called a tripled fixed point of the mapping $F: X \times X \times X \rightarrow X$ if

$$
F(x, y, z)=x, \quad F(y, z, x)=y \quad \text { and } \quad F(z, x, y)=z .
$$

Definition 2.3 A mapping $F: X \times X \times X \rightarrow X$ is said to be a Lipschitzian mapping if for each integer $n \geq 1$, there exists a constant $k_{n}>0$ such that

$$
d\left(F^{n}(x, y, z), F^{n}(u, v, w)\right) \leq \frac{k_{n}}{3}[d(x, u)+d(y, v)+d(z, w)] \quad \forall x, y, z \in X
$$

where $F^{n}(x, y, z)=F^{n-1}(F(x, y, z), F(y, z, x), F(z, x, y))$.

If $k_{n}=k \forall n \geq 1$, then $F$ is called uniformly Lipschitzian and if $k_{n}=1 \forall n \geq 1$, then $F$ is called nonexpansive.

Definition 2.4 A mapping $F: X \times X \times X \rightarrow X$ is called asymptotically regular if

$$
\lim _{n \rightarrow \infty} d\left(F^{n+1}(x, y, z), F^{n}(x, y, z)\right)=0 \quad \forall x, y, z \in X
$$


Let $G$ be a subsemigroup of $[0, \infty)$ with addition '+' such that

$$
t-s \in G \quad \forall t, s \in G \text { with } t \geq s \text {. }
$$

This condition is satisfied if $G=[0, \infty)$ or $G=Z^{+}$, the set of nonnegative integers. Let $\mathfrak{I}=\{F(t): t \in G\}$ be a family of self-mappings on $X \times X \times X$. Then $\mathfrak{s}$ is called a (oneparameter) semigroup on $X \times X \times X$ if the following conditions are satisfied:

(i) $F(0)(x, y, z)=x, F(0)(y, z, x)=y$ and $F(0)(z, x, y)=z \forall x, y, z \in X$;

(ii) $F(s)(F(t)(x, y, z), F(t)(y, z, x), F(t)(z, x, y))=F(s+t)(x, y, z) \forall s, t \in G$ and $x, y, z \in X$;

(iii) $\forall x, y, z \in X$, the self-mappings $t \mapsto F(t)(x, y, z), t \mapsto F(t)(y, z, x)$ and $t \mapsto F(t)(z, x, y)$ from $G$ into $X$ are continuous when $G$ has the relative topology of $[0, \infty)$.

A semigroup $\mathfrak{I}=\{F(t): t \in G\}$ on $X \times X \times X$ is said to be asymptotically regular at a point $(x, y, z) \in X \times X \times X$ if

$$
\lim _{t \rightarrow \infty} d(F(t+h)(x, y, z), F(t)(x, y, z))=0 \quad \forall h \in G
$$

If $\Im$ is asymptotically regular at each $(x, y, z) \in X \times X \times X$, then $\Im$ is called an asymptotically regular semigroup on $X \times X \times X$.

Definition 2.5 A semigroup $\mathfrak{\Im}=\{F(t): t \in G\}$ on $X \times X \times X$ is called a uniformly Lipschitzian semigroup if

$$
\sup \{k(t): t \in G\}=k<\infty
$$

where

$$
k(t)=3 \sup \left\{\frac{d(F(t)(x, y, z), F(t)(u, v, w))}{[d(x, u)+d(y, v)+d(z, w)] \neq 0}: x, y, z \in X\right\} .
$$

In a metric space $(X, d)$, let $\mu$ denote a nonempty family of subsets of $X$. Following Khamsi [11], we say that $\mu$ defines a convexity structure on $X$ if $\mu$ is stable under intersection. We say that $\mu$ has property $(R)$ if any decreasing sequence $\left\{C_{n}\right\}$ of nonempty bounded closed subsets of $X$ with $C_{n} \in \mu$ has a nonempty intersection. Recall that a subset of $X$ is said to be admissible [5] if it is an intersection of closed balls. We denote by $A(X)$ the family of all admissible subsets of $X$. It is obvious that $A(X)$ defines a convexity structure on $X$. In this paper any other convexity structure $\mu$ on $X$ is always assumed to contain $A(X)$.

Let $M$ be a bounded subset of $X$. Following Lim and Xu [13], we shall adopt the following notations:

$$
\begin{aligned}
& B(x, r) \text { is the closed ball centered at } x \text { with radius } r, \\
& r(x, M)=\sup \{d(x, y): y \in M\} \text { for } x \in X, \\
& \delta(M)=\sup \{r(x, M): x \in M\}, \\
& R(M)=\inf \{r(x, M): x \in M\} .
\end{aligned}
$$

For a bounded subset $A$ of $X$, we define the admissible hull of $A$, denoted by $\operatorname{ad}(A)$, as the intersection of all those admissible subsets of $X$ which contain $A$, i.e.,

$$
\operatorname{ad}(A)=\bigcap\{B: A \subseteq B \subseteq X \text { with } B \text { admissible }\} .
$$


Proposition 2.1 [13] For a point $x \in X$ and a bounded subset $A$ of $X$, we have

$$
r(x, a d(A))=r(x, A) .
$$

Definition 2.6 [11] A metric space $(X, d)$ is said to have normal (resp. uniform normal) structure if there exists a convexity structure $\mu$ on $X$ such that $R(A)<\delta(A)($ resp. $R(A) \leq$ $c \cdot \delta(A)$ for some constant $c \in(0,1))$ for all $A \in \mu$ which is bounded and consists of more than one point. In this case $\mu$ is said to be normal (resp. uniformly normal) in $X$.

We define the normal structure coefficient $\bar{N}(X)$ of $X$ (with respect to a given convexity structure $\mu$ ) as the number

$$
\sup \left\{\frac{R(A)}{\delta(A)}\right\}
$$

where the supremum is taken over all bounded $A \in F$ with $\delta(A)>0 . X$ then has uniform normal structure if and only if $\bar{N}(X)<1$.

Khamsi proved the following result that will be very useful in the proof of our main theorem.

Proposition 2.2 [11] Let $X$ be a complete bounded metric space and $\mu$ be a convexity structure of $X$ with uniform normal structure. Then $\mu$ has property $(R)$.

Definition 2.7 [14] Let $(X, d)$ be a metric space and $\Im=\{F(t): t \in G\}$ be a semigroup on $X \times X \times X$. Let us write the set

$$
w(\infty)=\left\{\left\{t_{n}\right\}:\left\{t_{n}\right\} \subset G \text { and } t_{n} \rightarrow \infty\right\} .
$$

Lemma 2.1 [14] If $\left\{t_{n}\right\} \in \omega(\infty)$, then $\left\{t_{n+1}-t_{n}\right\} \in \omega(\infty)$.

Definition 2.8 [13] A metric space $(X, d)$ is said to have property $(P)$ if given any two bounded sequences $\left\{x_{n}\right\}$ and $\left\{z_{n}\right\}$ in $X$, one can find some $z \in \bigcap_{n=1}^{\infty} a d\left\{z_{j}: j \geq n\right\}$ such that

$$
\limsup _{n \rightarrow \infty} d\left(z, x_{n}\right) \leq \limsup _{j \rightarrow \infty} \limsup _{n \rightarrow \infty} d\left(z_{j}, x_{n}\right)
$$

Definition 2.9 Let $(X, d)$ be a complete bounded metric space and $\Im=\{F(t): t \in G\}$ be a semigroup on $X \times X \times X$. Then $\Im$ has property $(*)$ if for each $x \in X$ and each $\left\{t_{n}\right\} \in w(\infty)$, the following conditions are satisfied:

(a) the sequences $\left\{F\left(t_{n}\right)(x, y, z)\right\},\left\{F\left(t_{n}\right)(y, z, x)\right\}$ and $\left\{F\left(t_{n}\right)(z, x, y)\right\}$ are bounded;

(b) for any sequence $\left\{s_{n}\right\}$ in $a d\left\{F\left(t_{n}\right)(x, y, z): n \geq 1\right\}$, there exists some $s \in \bigcap_{n=1}^{\infty} a d\left\{s_{j}: j \geq n\right\}$ such that

$$
\limsup _{n \rightarrow \infty} d\left(s, F\left(t_{n}\right)(x, y, z)\right) \leq \limsup _{j \rightarrow \infty} \limsup _{n \rightarrow \infty} d\left(s_{j}, F\left(t_{n}\right)(x, y, z)\right)
$$

for any sequence $\left\{u_{n}\right\}$ in $\operatorname{ad}\left\{F\left(t_{n}\right)(y, z, x): n \geq 1\right\}$, there exists some $u \in \bigcap_{n=1}^{\infty} a d\left\{u_{j}: j \geq n\right\}$ such that

$$
\limsup _{n \rightarrow \infty} d\left(u, F\left(t_{n}\right)(y, z, x)\right) \leq \limsup _{j \rightarrow \infty} \limsup _{n \rightarrow \infty} d\left(u_{j}, F\left(t_{n}\right)(y, z, x)\right)
$$


for any sequence $\left\{v_{n}\right\}$ in $\operatorname{ad}\left\{F\left(t_{n}\right)(z, x, y): n \geq 1\right\}$, there exists some $v \in \bigcap_{n=1}^{\infty} a d\left\{v_{j}: j \geq n\right\}$ such that

$$
\limsup _{n \rightarrow \infty} d\left(v, F\left(t_{n}\right)(z, x, y)\right) \leq \limsup _{j \rightarrow \infty} \limsup _{n \rightarrow \infty} d\left(v_{j}, F\left(t_{n}\right)(z, x, y)\right)
$$

Remark 2.1 If $X$ is a complete bounded metric space with property $(P)$, then each semigroup $\Im=\{F(t): t \in G\}$ on $X \times X \times X$ has property $(*)$.

\section{Main results}

Lemma 3.1 Let $(X, d)$ be a complete bounded metric space with uniform normal structure, and let $\Im=\{F(t): t \in G\}$ be a semigroup on $X \times X \times X$ with property $(*)$. Then, for each $x \in X$, each $\left\{t_{n}\right\} \in \omega(\infty)$ and for any constant $\tilde{N}(X)<c$, the normal structure coefficient with respect to the given convexity structure $\mu$, there exist some $s \in \bigcap_{n=1}^{\infty} a d\left\{s_{j}: j \geq n\right\}$, $u \in \bigcap_{n=1}^{\infty} a d\left\{u_{j}: j \geq n\right\}$ and $v \in \bigcap_{n=1}^{\infty} a d\left\{v_{j}: j \geq n\right\}$ satisfying the properties:

(I)

$$
\begin{aligned}
& \limsup _{n \rightarrow \infty} d\left(s, F\left(t_{n}\right)(x, y, z)\right) \leq \widehat{c} \cdot A\left(\left\{F\left(t_{n}\right)(x, y, z)\right\}\right), \\
& \limsup _{n \rightarrow \infty} d\left(u, F\left(t_{n}\right)(y, z, x)\right) \leq \widehat{c} \cdot B\left(\left\{F\left(t_{n}\right)(y, z, x)\right\}\right) \text { and } \\
& \limsup _{n \rightarrow \infty} d\left(v, F\left(t_{n}\right)(z, x, y)\right) \leq \widehat{c} \cdot C\left(\left\{F\left(t_{n}\right)(z, x, y)\right\}\right),
\end{aligned}
$$

where

$$
\begin{aligned}
& A\left(\left\{F\left(t_{n}\right)(x, y, z)\right\}\right)=\limsup _{n \rightarrow \infty}\left\{d\left(F\left(t_{i}\right)(x, y, z), F\left(t_{j}\right)(x, y, z)\right): i, j \geq n\right\}, \\
& B\left(\left\{F\left(t_{n}\right)(y, z, x)\right\}\right)=\limsup _{n \rightarrow \infty}\left\{d\left(F\left(t_{i}\right)(y, z, x), F\left(t_{j}\right)(y, z, x)\right): i, j \geq n\right\} \quad \text { and } \\
& C\left(\left\{F\left(t_{n}\right)(z, x, y)\right\}\right)=\limsup _{n \rightarrow \infty}\left\{d\left(F\left(t_{i}\right)(z, x, y), F\left(t_{j}\right)(z, x, y)\right): i, j \geq n\right\} ;
\end{aligned}
$$

(II)

$$
\begin{aligned}
& d(s, w) \leq \limsup _{n \rightarrow \infty} d\left(F\left(t_{n}\right)(x, y, z), w\right), \\
& d(u, w) \leq \limsup _{n \rightarrow \infty} d\left(F\left(t_{n}\right)(y, z, x), w\right) \quad \text { and } \\
& d(v, w) \leq \limsup _{n \rightarrow \infty} d\left(F\left(t_{n}\right)(z, x, y), w\right) \quad \text { for all } w \in X .
\end{aligned}
$$

Proof For each integer $n \geq 1$, let $A_{n}=\left\{F\left(t_{j}\right)(x, y, z): j \geq n\right\}, B_{n}=\left\{F\left(t_{j}\right)(y, z, x): j \geq n\right\}$ and $C_{n}=\left\{F\left(t_{j}\right)(z, x, y): j \geq n\right\}$. Then $\left\{A_{n}\right\},\left\{B_{n}\right\}$ and $\left\{C_{n}\right\}$ are decreasing sequences of admissible subsets of $X$, hence $A:=\bigcap_{n=1}^{\infty} A_{n} \neq \phi, B:=\bigcap_{n=1}^{\infty} B_{n} \neq \phi$ and $C:=\bigcap_{n=1}^{\infty} C_{n} \neq \phi$ by Proposition 2.1. From Proposition 2.1, it is not difficult to see that $\delta\left(A_{n}\right)=\delta\left(\left\{F\left(t_{i}\right)(x, y, z): i \geq n\right\}\right)$, $\delta\left(B_{n}\right)=\delta\left(\left\{F\left(t_{i}\right)(y, z, x): i \geq n\right\}\right)$ and $\delta\left(C_{n}\right)=\delta\left(\left\{F\left(t_{i}\right)(z, x, y): i \geq n\right\}\right)$. Indeed, observe that

$$
\begin{aligned}
\delta\left(A_{n}\right) & =\sup \left\{r\left(w, A_{n}\right): w \in A_{n}\right\}=\sup _{w \in A_{n}} \sup _{j \geq n} d\left(w, F\left(t_{j}\right)(x, y, z)\right) \\
& =\sup _{j \geq n} \sup _{w \in A_{n}} d\left(w, F\left(t_{j}\right)(x, y, z)\right)=\sup _{j \geq n} r\left(F\left(t_{j}\right)(x, y, z), A_{n}\right)
\end{aligned}
$$




$$
\begin{aligned}
& =\sup _{j \geq n} \sup _{i \geq n} d\left(F\left(t_{j}\right)(x, y, z), F\left(t_{i}\right)(x, y, z)\right) \\
& =\delta\left(\left\{F\left(t_{i}\right)(x, y, z): i \geq n\right\}\right) .
\end{aligned}
$$

Similarly, one can obtain

$$
\begin{aligned}
& \delta\left(B_{n}\right)=\delta\left(\left\{F\left(t_{i}\right)(y, z, x): i \geq n\right\}\right), \\
& \delta\left(C_{n}\right)=\delta\left(\left\{F\left(t_{i}\right)(z, x, y): i \geq n\right\}\right) .
\end{aligned}
$$

On the other hand, for any $a \in A$ and any $w \in X$, we have

$$
\sup _{j \geq n} d\left(w, F\left(t_{j}\right)(x, y, z)\right)=r\left(w, A_{n}\right) \geq r(w, A) \geq d(w, a) .
$$

Therefore,

$$
d(w, a) \leq \limsup _{n \rightarrow \infty} d\left(w, F\left(t_{n}\right)(x, y, z)\right)
$$

Also, one can deduce that for any $b \in B, c \in C$ and any $w \in X$, we have

$$
\begin{aligned}
& d(w, b) \leq \limsup _{n \rightarrow \infty} d\left(w, F\left(t_{n}\right)(y, z, x)\right), \\
& d(w, c) \leq \limsup _{n \rightarrow \infty} d\left(w, F\left(t_{n}\right)(z, x, y)\right),
\end{aligned}
$$

from which (ii) follows.

We now claim that for each $n \geq 1$, there exist $a_{n} \in A_{n}, b_{n} \in B_{n}$ and $c_{n} \in C_{n}$ such that

$$
\begin{aligned}
& r\left(a_{n}, A_{n}\right) \leq \widehat{c} \cdot \delta\left(\left\{F\left(t_{j}\right)(x, y, z): j \geq n\right\}\right), \\
& r\left(b_{n}, B_{n}\right) \leq \widehat{c} \cdot \delta\left(\left\{F\left(t_{j}\right)(y, z, x): j \geq n\right\}\right), \\
& r\left(c_{n}, C_{n}\right) \leq \widehat{c} \cdot \delta\left(\left\{F\left(t_{j}\right)(z, x, y): j \geq n\right\}\right) .
\end{aligned}
$$

Indeed, if $\delta\left(\left\{F\left(t_{j}\right)(x, y, z): j \geq n\right\}\right)=0$, then $\delta\left(A_{n}\right)=\delta\left(\left\{F\left(t_{j}\right)(x, y, z): j \geq n\right\}\right)$, we conclude that (3) holds. Without loss of generality, we may assume that $\delta\left(\left\{F\left(t_{j}\right)(x, y, z): j \geq 0\right\}\right)>0$. Then, for $c>N(X)$, we choose $\epsilon>0$ so small satisfying the following:

$$
N(X) \delta\left(\left\{F\left(t_{j}\right)(x, y, z): j \geq n\right\}\right)+\epsilon \leq \widehat{c} \cdot \delta\left(\left\{F\left(t_{j}\right)(x, y, z): j \geq n\right\}\right)
$$

By the definition of $R\left(A_{n}\right)$, one can find $u_{n} \in A_{n}$ such that

$$
\begin{aligned}
r\left(u_{n}, A_{n}\right) & <R\left(A_{n}\right)+\epsilon \leq N(X) \delta\left(A_{n}\right)+\epsilon \\
& =N(X) \delta\left(\left\{F\left(t_{j}\right)(x, y, z): j \geq n\right\}\right)+\epsilon \\
& \leq \widehat{c} \cdot \delta\left(\left\{F\left(t_{j}\right)(x, y, z): j \geq n\right\}\right) .
\end{aligned}
$$

This shows that (3) holds. Obviously, it follows from (3) that for each $n \geq 1$,

$$
\limsup _{j \rightarrow \infty} r\left(u_{n}, x_{j}\right) \leq \widehat{c} \cdot \delta\left(\left\{F\left(t_{j}\right)(x, y, z): j \geq n\right\}\right)
$$


which implies

$$
\limsup _{n \rightarrow \infty} \limsup _{j \rightarrow \infty} r\left(u_{n}, F\left(t_{j}(x, y, z)\right)\right) \leq \widehat{c} \cdot A\left(\left\{F\left(t_{n}\right)(x, y, z)\right\}\right),
$$

where $A\left(\left\{F\left(t_{n}\right)(x, y, z)\right\}\right)=\left\{d\left(F\left(t_{j}\right)(x, y, z), F\left(t_{i}\right)(x, y, z)\right): i, j \geq n\right\}$. Noticing

$$
u_{n} \in A_{n} \subset \operatorname{ad}\left\{F\left(t_{j}\right)(x, y, z): j \geq n\right\} \quad \text { for each } n \geq 1 \text {, }
$$

we know that property (*) yields a point $s \in \bigcap_{n=1}^{\infty} a d\left\{s_{j}: j \geq n\right\}$ such that

$$
\limsup _{j \rightarrow \infty} d\left(s, F\left(t_{j}\right)(x, y, z)\right) \leq \limsup _{n \rightarrow \infty} \limsup _{j \rightarrow \infty} r\left(s_{n}, F\left(t_{j}\right)(x, y, z)\right) .
$$

Since $\left\{s_{j}: j \geq n\right\} \subset A_{n}, s \in A=\bigcap_{n=1}^{\infty} a d\left\{F\left(t_{j}\right)(x, y, z): j \geq n\right\}$ and satisfies

$$
\limsup _{j \rightarrow \infty} d\left(s, F\left(t_{j}\right)(x, y, z)\right) \leq \widehat{c} \cdot A\left(\left\{F\left(t_{j}\right)(x, y, z)\right\}\right), \quad \text { by }(7)
$$

similarly one can obtain that

$$
\begin{aligned}
& \limsup _{j \rightarrow \infty} d\left(u, F\left(t_{j}\right)(y, z, x)\right) \leq \widehat{c} \cdot B\left(\left\{F\left(t_{j}\right)(y, z, x)\right\}\right), \\
& \limsup _{j \rightarrow \infty} d\left(v, F\left(t_{j}\right)(z, x, y)\right) \leq \widehat{c} \cdot C\left(\left\{F\left(t_{j}\right)(z, x, y)\right\}\right),
\end{aligned}
$$

where $u \in B=\bigcap_{n=1}^{\infty} a d\left\{F\left(t_{j}\right)(y, z, x): j \geq n\right\}$ and $v \in C=\bigcap_{n=1}^{\infty} a d\left\{F\left(t_{j}\right)(z, x, y): j \geq n\right\}$.

Therefore (i) holds.

Theorem 3.1 Let $(X, d)$ be a complete bounded metric space with uniform normal structure, and let $\mathfrak{s}=\{F(t): t \in G\}$ be an asymptotically regular semigroup on $X \times X \times X$ with property $(*)$ and satisfying

$$
\left(\liminf _{t \rightarrow \infty} k(t)\right) \cdot\left(\limsup _{t \rightarrow \infty} k(t)\right)<\bar{N}(X)^{-\frac{1}{2}}
$$

Then there exist some $x, y, z \in X$ such that $F(t)(x, y, z)=x, F(t)(y, z, x)=y$ and $F(t)(z, x, y)=z$ for all $t \in G$.

Proof First, we write $k=\liminf _{t \rightarrow \infty} k(t)$ and $\widetilde{k}=\lim \sup _{t \rightarrow \infty} k(t)$. Choose a constant $\widehat{c}$ such that $\tilde{N}(X)<\widehat{c}<1$ and $k \widetilde{k}<\frac{1}{\sqrt{c}}$. We can select a sequence $\left\{t_{n}\right\} \in w(\infty)$ such that $\left\{t_{n+1}-t_{n}\right\} \in$ $w(\infty)$ and $\lim _{n \rightarrow \infty} k\left(t_{n}\right)=\widetilde{k}$, where $\widetilde{k}>0$.

Observe that

$$
\left\{d\left(F\left(t_{j}\right)(x, y, z), F\left(t_{i}\right)(x, y, z)\right): i, j \geq n\right\}=\left\{d\left(F\left(t_{j}\right)(x, y, z), F\left(t_{i}\right)(x, y, z)\right): j>i \geq n\right\} \cup\{0\}
$$

for each $n \in N$ and $x, y, z \in X$. 
Now fix $x_{0}, y_{0}, z_{0} \in X$. Then, by Lemma 3.1, we can inductively construct sequences $\left\{x_{l}\right\}_{l=1}^{\infty},\left\{y_{l}\right\}_{l=1}^{\infty},\left\{z_{l}\right\}_{l=1}^{\infty} \subset X$ such that

$$
\begin{aligned}
& x_{l+1} \in \bigcap_{n=1}^{\infty} a d\left\{F\left(t_{i}\right)\left(x_{l}, y_{l}, z_{l}\right): i \geq n\right\}, \quad y_{l+1} \in \bigcap_{n=1}^{\infty} a d\left\{F\left(t_{i}\right)\left(y_{l}, z_{l}, x_{l}\right): i \geq n\right\} \quad \text { and } \\
& z_{l+1} \in \bigcap_{n=1}^{\infty} a d\left\{F\left(t_{i}\right)\left(z_{l}, x_{l}, y_{l}\right): i \geq n\right\}, \quad \text { for each integer } l \geq 0,
\end{aligned}
$$

(III)

$$
\begin{aligned}
& \limsup _{n \rightarrow \infty} d\left(F\left(t_{n}\right)\left(x_{l}, y_{l}, z_{l}\right), x_{l+1}\right) \leq \widehat{c} \cdot A\left(\left\{F\left(t_{n}\right)\left(x_{l}, y_{l}, z_{l}\right)\right\}\right), \\
& \limsup _{n \rightarrow \infty} d\left(F\left(t_{n}\right)\left(y_{l}, z_{l}, x_{l}\right), y_{l+1}\right) \leq \widehat{c} \cdot B\left(\left\{F\left(t_{n}\right)\left(y_{l}, z_{l}, x_{l}\right)\right\}\right), \\
& \limsup _{n \rightarrow \infty} d\left(F\left(t_{n}\right)\left(z_{l}, x_{l}, y_{l}\right), z_{l+1}\right) \leq \widehat{c} \cdot C\left(\left\{F\left(t_{n}\right)\left(z_{l}, x_{l}, y_{l}\right)\right\}\right),
\end{aligned}
$$

where

$$
\begin{aligned}
& A\left(\left\{F\left(t_{n}\right)\left(x_{l}, y_{l}, z_{l}\right)\right\}\right)=\limsup _{n \rightarrow \infty}\left\{d\left(F\left(t_{i}\right)\left(x_{l}, y_{l}, z_{l}\right), F\left(t_{j}\right)\left(x_{l}, y_{l}, z_{l}\right)\right): i, j \geq n\right\}, \\
& B\left(\left\{F\left(t_{n}\right)\left(y_{l}, z_{l}, x_{l}\right)\right\}\right)=\limsup _{n \rightarrow \infty}\left\{d\left(F\left(t_{i}\right)\left(y_{l}, z_{l}, x_{l}\right), F\left(t_{j}\right)\left(y_{l}, z_{l}, x_{l}\right)\right): i, j \geq n\right\} \quad \text { and } \\
& C\left(\left\{F\left(t_{n}\right)\left(z_{l}, x_{l}, y_{l}\right)\right\}\right)=\limsup _{n \rightarrow \infty}\left\{d\left(F\left(t_{i}\right)\left(z_{l}, x_{l}, y_{l}\right), F\left(t_{j}\right)\left(z_{l}, x_{l}, y_{l}\right)\right): i, j \geq n\right\} ;
\end{aligned}
$$

(IV)

$$
\begin{aligned}
& d\left(x_{l+1}, w\right) \leq \limsup _{n \rightarrow \infty} d\left(F\left(t_{n}\right)\left(x_{l}, y_{l}, z_{l}\right), w\right), \\
& d\left(y_{l+1}, w\right) \leq \limsup _{n \rightarrow \infty} d\left(F\left(t_{n}\right)\left(y_{l}, z_{l}, x_{l}\right), w\right) \quad \text { and } \\
& d\left(z_{l+1}, w\right) \leq \limsup _{n \rightarrow \infty} d\left(F\left(t_{n}\right)\left(z_{l}, x_{l}, y_{l}\right), w\right) \quad \forall w \in X .
\end{aligned}
$$

Let

$$
\begin{aligned}
& D_{l}=\limsup _{n \rightarrow \infty}\left[d\left(x_{l+1}, F\left(t_{n}\right)\left(x_{l}, y_{l}, z_{l}\right)\right)+d\left(y_{l+1}, F\left(t_{n}\right)\left(y_{l}, z_{l}, x_{l}\right)\right)\right. \\
& \left.+d\left(z_{l+1}, F\left(t_{n}\right)\left(z_{l}, x_{l}, y_{l}\right)\right)\right] \text { and } \\
& h=\widehat{c} \cdot k \widetilde{k}<1 \text {. }
\end{aligned}
$$

Observe that for each $i>j \geq 1$, using (IV) we have

$$
\begin{aligned}
& d\left(F\left(t_{i}\right)\left(x_{l}, y_{l}, z_{l}\right), F\left(t_{j}\right)\left(x_{l}, y_{l}, z_{l}\right)\right) \\
& \quad=d\left(F\left(t_{j}\right)\left(x_{l}, y_{l}, z_{l}\right), F\left(t_{j}\right) F\left(t_{i}-t_{j}\right)\left(x_{l}, y_{l}, z_{l}\right)\right) \\
& \quad=d\left(F\left(t_{j}\right)\left(x_{l}, y_{l}, z_{l}\right), F\left(t_{j}\right)\left(F\left(t_{i}-t_{j}\right)\left(x_{l}, y_{l}, z_{l}\right), F\left(t_{i}-t_{j}\right)\left(y_{l}, z_{l}, x_{l}\right), F\left(t_{i}-t_{j}\right)\left(z_{l}, x_{l}, y_{l}\right)\right)\right) \\
& \quad \leq \frac{k\left(t_{j}\right)}{3}\left[d\left(x_{l}, F\left(t_{i}-t_{j}\right)\left(x_{l}, y_{l}, z_{l}\right)\right)+d\left(y_{l}, F\left(t_{i}-t_{j}\right)\left(y_{l}, z_{l}, x_{l}\right)\right)\right.
\end{aligned}
$$




$$
\begin{aligned}
& \left.+d\left(z_{l}, F\left(t_{i}-t_{j}\right)\left(z_{l}, x_{l}, y_{l}\right)\right)\right] \\
\leq & \frac{k\left(t_{j}\right)}{3} \limsup _{n \rightarrow \infty}\left[d\left(F\left(t_{n}\right)\left(x_{l-1}, y_{l-1}, z_{l-1}\right), F\left(t_{i}-t_{j}\right)\left(x_{l}, y_{l}, z_{l}\right)\right)\right. \\
& +d\left(F\left(t_{n}\right)\left(y_{l-1}, z_{l-1}, x_{l-1}\right), F\left(t_{i}-t_{j}\right)\left(y_{l}, z_{l}, x_{l}\right)\right) \\
& \left.+d\left(F\left(t_{n}\right)\left(z_{l-1}, x_{l-1}, y_{l-1}\right), F\left(t_{i}-t_{j}\right)\left(z_{l}, x_{l}, y_{l}\right)\right)\right] .
\end{aligned}
$$

By the asymptotic regularity of $\Im=\{F(t): t \in G\}$ on $X \times X \times X$, we see that

$$
\begin{aligned}
& \limsup _{n \rightarrow \infty}\left[d\left(F\left(t_{n}\right)\left(x_{l-1}, y_{l-1}, z_{l-1}\right), F\left(t_{n}+t_{i}-t_{j}\right)\left(x_{l-1}, y_{l-1}, z_{l-1}\right)\right)\right]=0 \\
& \limsup _{n \rightarrow \infty}\left[d\left(F\left(t_{n}\right)\left(y_{l-1}, z_{l-1}, x_{l-1}\right), F\left(t_{n}+t_{i}-t_{j}\right)\left(y_{l-1}, z_{l-1}, x_{l-1}\right)\right)\right]=0 \quad \text { and } \\
& \limsup _{n \rightarrow \infty}\left[d\left(F\left(t_{n}\right)\left(z_{l-1}, x_{l-1}, y_{l-1}\right), F\left(t_{n}+t_{i}-t_{j}\right)\left(z_{l-1}, x_{l-1}, y_{l-1}\right)\right)\right]=0
\end{aligned}
$$

which implies

$$
\begin{aligned}
& \limsup _{n \rightarrow \infty} d\left(F\left(t_{n}\right)\left(x_{l-1}, y_{l-1}, z_{l-1}\right), F\left(t_{i}-t_{j}\right)\left(x_{l}, y_{l}, z_{l}\right)\right) \\
& \leq \limsup _{n \rightarrow \infty} d\left(F\left(t_{n}\right)\left(x_{l-1}, y_{l-1}, z_{l-1}\right), F\left(t_{n}+t_{i}-t_{j}\right)\left(x_{l-1}, y_{l-1}, z_{l-1}\right)\right) \\
& \quad+\limsup _{n \rightarrow \infty} d\left(F\left(t_{n}+t_{i}-t_{j}\right)\left(x_{l-1}, y_{l-1}, z_{l-1}\right), F\left(t_{i}-t_{j}\right)\left(x_{l}, y_{l}, z_{l}\right)\right) \\
& \leq \limsup _{n \rightarrow \infty} d\left(F ( t _ { i } - t _ { j } ) \left(F\left(t_{n}\right)\left(x_{l-1}, y_{l-1}, z_{l-1}\right), F\left(t_{n}\right)\left(y_{l-1}, z_{l-1}, x_{l-1}\right),\right.\right. \\
&\left.\left.\quad F\left(t_{n}\right)\left(z_{l-1}, x_{l-1}, y_{l-1}\right)\right), F\left(t_{i}-t_{j}\right)\left(x_{l}, y_{l}, z_{l}\right)\right) \\
& \leq \\
& \quad \frac{k\left(t_{i}-t_{j}\right)}{3}\left[d\left(F\left(t_{n}\right)\left(x_{l-1}, y_{l-1}, z_{l-1}\right), x_{l}\right)+d\left(F\left(t_{n}\right)\left(y_{l-1}, z_{l-1}, x_{l-1}\right), y_{l}\right)\right. \\
&\left.\quad+d\left(F\left(t_{n}\right)\left(z_{l-1}, x_{l-1}, y_{l-1}\right), z_{l}\right)\right] \\
& \leq \frac{k\left(t_{i}-t_{j}\right)}{3} D_{l-1} .
\end{aligned}
$$

Similarly, one can show that

$$
\begin{aligned}
& \limsup _{n \rightarrow \infty} d\left(F\left(t_{n}\right)\left(y_{l-1}, z_{l-1}, x_{l-1}\right), F\left(t_{i}-t_{j}\right)\left(y_{l}, z_{l}, x_{l}\right)\right) \leq \frac{k\left(t_{i}-t_{j}\right)}{3} D_{l-1}, \\
& \limsup _{n \rightarrow \infty} d\left(F\left(t_{n}\right)\left(z_{l-1}, x_{l-1}, y_{l-1}\right), F\left(t_{i}-t_{j}\right)\left(z_{l}, x_{l}, y_{l}\right)\right) \leq \frac{k\left(t_{i}-t_{j}\right)}{3} D_{l-1} .
\end{aligned}
$$

Then it follows from (10), (11) and (12) that for each $i>j \geq 1$,

$$
d\left(F\left(t_{i}\right)\left(x_{l}, y_{l}, z_{l}\right), F\left(t_{j}\right)\left(x_{l}, y_{l}, z_{l}\right)\right) \leq \frac{k\left(t_{j}\right)}{3} \cdot k\left(t_{i}-t_{j}\right) \cdot D_{l-1},
$$

which implies that for each $n \geq 1$,

$$
\begin{aligned}
& \sup \left\{d\left(F\left(t_{i}\right)\left(x_{l}, y_{l}, z_{l}\right), F\left(t_{j}\right)\left(x_{l}, y_{l}, z_{l}\right)\right): i, j \geq n\right\} \\
& \quad=\sup \left\{d\left(F\left(t_{i}\right)\left(x_{l}, y_{l}, z_{l}\right), F\left(t_{j}\right)\left(x_{l}, y_{l}, z_{l}\right)\right): i>j \geq n\right\}
\end{aligned}
$$




$$
\begin{aligned}
& \leq \sup \left\{\frac{k\left(t_{j}\right)}{3} \cdot k\left(t_{i}-t_{j}\right) \cdot D_{l-1}: i>j \geq n\right\} \\
& \leq \frac{D_{l-1}}{3} \cdot \sup \left\{k\left(t_{j}\right): j \geq n\right\} \cdot \sup \left\{k\left(t_{i}-t_{j}\right): i>j \geq n\right\} \\
& \leq \frac{D_{l-1}}{3} \cdot \sup \left\{k\left(t_{j}\right): j \geq n\right\} \cdot \sup \left\{k(t): G \ni t \geq t_{n+1}-t_{n}\right\} .
\end{aligned}
$$

Hence, by using (III) and (9), we have

$$
\begin{aligned}
& D_{l}= \limsup _{n \rightarrow \infty}\left[d\left(x_{l+1}, F\left(t_{n}\right)\left(x_{l}, y_{l}, z_{l}\right)\right)+d\left(y_{l+1}, F\left(t_{n}\right)\left(y_{l}, z_{l}, x_{l}\right)\right)+d\left(z_{l+1}, F\left(t_{n}\right)\left(z_{l}, x_{l}, y_{l}\right)\right)\right] \\
& \leq \widehat{c} \cdot\left[A\left(\left\{F\left(t_{n}\right)\left(x_{l}, y_{l}, z_{l}\right)\right\}\right)+B\left(\left\{F\left(t_{n}\right)\left(y_{l}, z_{l}, x_{l}\right)\right\}\right)+C\left(\left\{F\left(t_{n}\right)\left(z_{l}, x_{l}, y_{l}\right)\right\}\right)\right] \\
& \leq \widehat{c} \cdot \limsup _{n \rightarrow \infty}\left\{d\left(F\left(t_{i}\right)\left(x_{l}, y_{l}, z_{l}\right), F\left(t_{j}\right)\left(x_{l}, y_{l}, z_{l}\right)\right)+d\left(F\left(t_{i}\right)\left(y_{l}, z_{l}, x_{l}\right), F\left(t_{j}\right)\left(y_{l}, z_{l}, x_{l}\right)\right)\right. \\
&\left.+d\left(F\left(t_{i}\right)\left(z_{l}, x_{l}, y_{l}\right), F\left(t_{j}\right)\left(z_{l}, x_{l}, y_{l}\right)\right): i, j \geq n\right\} \\
& \leq \widehat{c} \cdot D_{l-1} \cdot \limsup _{n \rightarrow \infty} k\left(t_{n}\right) \cdot \limsup _{n \rightarrow \infty}\left\{k(t): G \ni t \geq t_{n+1}-t_{n}\right\} \\
& \leq \widehat{c} \cdot k \widetilde{k} \cdot D_{l-1} \leq h D_{l-1} \leq h^{2} D_{l-2} \leq \ldots \\
&= h^{l} D_{0} .
\end{aligned}
$$

Hence, by the asymptotic regularity of $\Im$ on $X \times X \times X$, we have, for each integer $n \geq 1$,

$$
\begin{aligned}
d\left(x_{l+1}, x_{l}\right) \leq & d\left(x_{l+1}, F\left(t_{n}\right)\left(x_{l}, y_{l}, z_{l}\right)\right)+d\left(x_{l}, F\left(t_{n}\right)\left(x_{l}, y_{l}, z_{l}\right)\right) \\
\leq & d\left(x_{l+1}, F\left(t_{n}\right)\left(x_{l}, y_{l}, z_{l}\right)\right)+\limsup _{m \rightarrow \infty} d\left(F\left(t_{m}\right)\left(x_{l-1}, y_{l-1}, z_{l-1}\right), F\left(t_{n}\right)\left(x_{l}, y_{l}, z_{l}\right)\right) \\
\leq & d\left(x_{l+1}, F\left(t_{n}\right)\left(x_{l}, y_{l}, z_{l}\right)\right)+\limsup _{m \rightarrow \infty} d\left(F\left(t_{m}\right)\left(x_{l-1}, y_{l-1}, z_{l-1}\right),\right. \\
& \left.F\left(t_{m}+t_{n}\right)\left(x_{l-1}, y_{l-1}, z_{l-1}\right)\right) \\
& +\limsup _{m \rightarrow \infty} d\left(F\left(t_{m}+t_{n}\right)\left(x_{l-1}, y_{l-1}, z_{l-1}\right), F\left(t_{n}\right)\left(x_{l}, y_{l}, z_{l}\right)\right) \\
\leq & d\left(x_{l+1}, F\left(t_{n}\right)\left(x_{l}, y_{l}, z_{l}\right)\right)+\frac{k\left(t_{n}\right)}{3} \cdot \limsup _{m \rightarrow \infty}\left[d\left(x_{l}, F\left(t_{m}\right)\left(x_{l-1}, y_{l-1}, z_{l-1}\right)\right)\right. \\
& \left.+d\left(y_{l}, F\left(t_{m}\right)\left(y_{l-1}, z_{l-1}, x_{l-1}\right)\right)+d\left(z_{l}, F\left(t_{m}\right)\left(z_{l-1}, x_{l-1}, y_{l-1}\right)\right)\right] \\
\leq & d\left(x_{l+1}, F\left(t_{n}\right)\left(x_{l}, y_{l}, z_{l}\right)\right)+\frac{k\left(t_{n}\right)}{3} \cdot D_{l-1},
\end{aligned}
$$

which implies

$$
\begin{aligned}
d\left(x_{l+1}, x_{l}\right) \leq & \limsup _{n \rightarrow \infty}\left[d\left(x_{l+1}, F\left(t_{n}\right)\left(x_{l}, y_{l}, z_{l}\right)\right)+d\left(y_{l+1}, F\left(t_{n}\right)\left(y_{l}, z_{l}, x_{l}\right)\right)\right. \\
& \left.+d\left(z_{l+1}, F\left(t_{n}\right)\left(z_{l}, x_{l}, y_{l}\right)\right)\right]+\frac{1}{3} D_{l-1} \cdot \limsup _{n \rightarrow \infty} k\left(t_{n}\right) \\
\leq & D_{l}+\frac{1}{3} \cdot k \cdot D_{l-1} .
\end{aligned}
$$

It follows from (14) that

$$
d\left(x_{l+1}, x_{l}\right) \leq D_{l}+\frac{1}{3} \cdot k \cdot D_{l-1} \leq\left(h^{l}+\frac{1}{3} k h^{l-1}\right) D_{0} \leq h^{l-1} \cdot \frac{2}{3} D_{0} \max \{h, k\} .
$$


Similarly, one can deduce that

$$
\begin{aligned}
& d\left(y_{l+1}, y_{l}\right) \leq h^{l-1} \cdot \frac{2}{3} D_{0} \max \{h, k\} \\
& d\left(z_{l+1}, z_{l}\right) \leq h^{l-1} \cdot \frac{2}{3} D_{0} \max \{h, k\} .
\end{aligned}
$$

Thus, we have $\sum_{l=0}^{\infty} d\left(x_{l+1}, x_{l}\right) \leq \frac{2}{3} D_{0} \max \{h, k\} \sum_{l=0}^{\infty} h^{l-1}<\infty, \sum_{l=0}^{\infty} d\left(y_{l+1}, y_{l}\right)<\infty$ and $\sum_{l=0}^{\infty} d\left(z_{l+1}, z_{l}\right)<\infty$. Consequently, $\left\{x_{l}\right\},\left\{y_{l}\right\}$ and $\left\{z_{l}\right\}$ are Cauchy and hence convergent as $X$ is complete. Let $x=\lim _{l \rightarrow \infty} x_{l}, y=\lim _{l \rightarrow \infty} y_{l}$ and $z=\lim _{l \rightarrow \infty} z_{l}$. Then we have

$$
\begin{aligned}
& \lim _{l \rightarrow \infty} d\left(F(s)\left(x_{l}, y_{l}, z_{l}\right), F(s)(x, y, z)\right)=0, \\
& \lim _{l \rightarrow \infty} d\left(F(s)\left(y_{l}, z_{l}, x_{l}\right), F(s)(y, z, x)\right)=0, \\
& \lim _{l \rightarrow \infty} d\left(F(s)\left(z_{l}, x_{l}, y_{l}\right), F(s)(z, x, y)\right)=0 .
\end{aligned}
$$

On the other hand, from (15) we have actually proven the following inequalities:

$$
\begin{aligned}
& \limsup _{n \rightarrow \infty} d\left(F\left(t_{n}\right)\left(x_{l}, y_{l}, z_{l}\right), x_{l}\right) \leq \frac{k\left(t_{n}\right)}{3} \cdot D_{l-1} \leq \frac{1}{3} k\left(t_{n}\right) h^{l-1} D_{0}, \\
& \limsup _{n \rightarrow \infty} d\left(F\left(t_{n}\right)\left(y_{l}, z_{l}, x_{l}\right), y_{l}\right) \leq \frac{1}{3} k\left(t_{n}\right) h^{l-1} D_{0}, \\
& \limsup _{n \rightarrow \infty} d\left(F\left(t_{n}\right)\left(z_{l}, x_{l}, y_{l}\right), z_{l}\right) \leq \frac{1}{3} k\left(t_{n}\right) h^{l-1} D_{0} .
\end{aligned}
$$

Since $\lim _{n \rightarrow \infty} k\left(t_{n}\right)=k$, it follows that

$$
\begin{aligned}
\limsup _{n \rightarrow \infty} d\left(x, F\left(t_{n}\right)(x, y, z)\right)= & d\left(x, x_{l}\right)+\limsup _{n \rightarrow \infty} d\left(x_{l}, F\left(t_{n}\right)\left(x_{l}, y_{l}, z_{l}\right)\right) \\
& +\limsup _{n \rightarrow \infty} d\left(F\left(t_{n}\right)\left(x_{l}, y_{l}, z_{l}\right), F\left(t_{n}\right)\left(x, y, z_{l}\right)\right) \\
\leq & d\left(x, x_{l}\right)+\frac{1}{3} \limsup _{n \rightarrow \infty} k\left(t_{n}\right) h^{l-1} D_{0} \\
\leq & d\left(x, x_{l}\right)+\frac{1}{3} k h^{l-1} D_{0} \rightarrow 0, \quad l \rightarrow \infty .
\end{aligned}
$$

Similarly, one can obtain that

$$
\begin{aligned}
& \limsup _{n \rightarrow \infty} d\left(y, F\left(t_{n}\right)(y, z, x)\right) \leq d\left(y, y_{l}\right)+\frac{1}{3} k h^{l-1} D_{0} \rightarrow 0, \quad l \rightarrow \infty, \\
& \limsup _{n \rightarrow \infty} d\left(z, F\left(t_{n}\right)(z, x, y)\right) \leq d\left(z, z_{l}\right)+\frac{1}{3} k h^{l-1} D_{0} \rightarrow 0, \quad l \rightarrow \infty,
\end{aligned}
$$

i.e., $\lim _{n \rightarrow \infty} d\left(x, F\left(t_{n}\right)(x, y, z)\right)=0, \lim _{n \rightarrow \infty} d\left(y, F\left(t_{n}\right)(y, z, x)\right)=0$ and $\lim _{n \rightarrow \infty} d\left(z, F\left(t_{n}\right)(z\right.$, $x, y))=0$. Hence, for each $s \in G$, by the continuity of $F(s)$, we deduce

$$
\begin{aligned}
d(x, F(s)(x, y, z)) & =\lim _{l \rightarrow \infty} d\left(x_{l}, F(s)\left(x_{l}, y_{l}, z_{l}\right)\right) \\
& \leq \lim _{l \rightarrow \infty} \limsup _{n \rightarrow \infty} d\left(x_{l}, F\left(t_{n}+s\right)\left(x_{l-1}, y_{l-1}, z_{l-1}\right)\right)
\end{aligned}
$$




$$
\begin{aligned}
\leq & \lim _{l \rightarrow \infty} \limsup _{n \rightarrow \infty} d\left(x_{l}, F\left(t_{n}\right)\left(x_{l-1}, y_{l-1}, z_{l-1}\right)\right) \\
& +\lim _{l \rightarrow \infty} \limsup _{n \rightarrow \infty} d\left(F\left(t_{n}\right)\left(x_{l-1}, y_{l-1}, z_{l-1}\right), F\left(t_{n}+s\right)\left(x_{l-1}, y_{l-1}, z_{l-1}\right)\right) \\
\leq & \lim _{l \rightarrow \infty} D_{l-1} \leq \lim _{l \rightarrow \infty} h^{l-1} D_{0}=0 .
\end{aligned}
$$

Similarly, we get that

$$
\begin{aligned}
& d(y, F(s)(y, z, x)) \leq \lim _{l \rightarrow \infty} D_{l-1} \leq \lim _{l \rightarrow \infty} h^{l-1} D_{0}=0, \\
& d(z, F(s)(z, x, y)) \leq \lim _{l \rightarrow \infty} D_{l-1} \leq \lim _{l \rightarrow \infty} h^{l-1} D_{0}=0 .
\end{aligned}
$$

Then we have $d(x, F(s)(x, y, z))=0, d(y, F(s)(y, z, x))=0$ and $d(z, F(s)(z, x, y))=0$, i.e., $F(s)(x, y, z)=x, F(s)(y, z, x)=y$ and $F(s)(z, x, y)=z$ for each $s \in G$.

From Remark 2.1 and Theorem 3.1, we immediately obtain the following results.

Corollary 3.1 Let $(X, d)$ be a complete bounded metric space with property $(P)$ and uniform normal structure, and let $\Im=\{F(t): t \in G\}$ be an asymptotically regular semigroup on $X \times X \times X$ satisfying

$$
\left(\liminf _{t \rightarrow \infty} k(t)\right) \cdot\left(\limsup _{t \rightarrow \infty} k(t)\right)<\bar{N}(X)^{-\frac{1}{2}}
$$

Then there exist some $x, y, z \in X$ such that $F(s)(x, y, z)=x, F(s)(y, z, x)=y$ and $F(s)(z, x, y)=z$ for all $t \in G$.

Remark 3.1 It will be interesting to establish Theorem 3.1 for representative $\psi=\{F(s)$ : $s \in S\}$ on $X \times X \times X$ of a left amenable semigroup $S$ as a complete bounded metric space with uniform normal structure as in Holmes and Lau [16], Lau and Takahashi [17] and Lau [18].

\section{Competing interests}

The author declares that they have no competing interests.

\section{Acknowledgements}

This project was funded by the Deanship of Scientific Research (DSR), King Khaled university, under project No. (KKU_S028_33). Also, the author is grateful to an anonymous referee for his fruitful comments.

Received: 15 May 2013 Accepted: 9 December 2013 Published: 17 Dec 2013

\section{References}

1. Boyd, DW, Wong, JS: On nonlinear contractions. Proc. Am. Math. Soc. 20, 458-464 (1969)

2. Casini, E, Maluta, E: Fixed points of uniformly Lipschitzian mappings in metric spaces with uniform normal structure. Nonlinear Anal. 9, 103-108 (1985)

3. Ciric, LB: A generalization of Banach's contraction principle. Proc. Am. Math. Soc. 45, 267-273 (1974)

4. Dorić, D, Kadelburg, Z, Radenović, S: Coupled fixed point results for mappings without mixed monotone property. Appl. Math. Lett. (2012). doi:10.1016/j.aml.2012.02.022

5. Dunford, N, Schwartz, JT: Linear Operators. Interscience, New York (1958)

6. Bhaskar, TG, Lakshmikantham, V: Fixed point theorems in partially ordered metric spaces and applications. Nonlinear Anal. 65, 1379-1393 (2006)

7. Imdad, M, Soliman, AH, Choudhury, BS, Das, P: On n-tupled coincidence point results in metric spaces. J. Oper. 2013, Article ID 532867 (2013)

8. Lakshmikantham, V, Ciric, L: Coupled fixed point theorems for nonlinear contractions in partially ordered metric spaces. Nonlinear Anal. 70, 4341-4349 (2009) 
9. Sabetghadam, F, Masiha, HP, Sanatpour, AH: Some coupled fixed point theorems in cone metric spaces. Fixed Point Theory Appl. 2009, Article ID 125426 (2009)

10. Samet, B, Vetro, C: Coupled fixed point, F-invariant set fixed point of N-order. Ann. Funct. Anal. 1, 46-56 (2010)

11. Khamsi, MA: On metric spaces with uniform normal structure. Proc. Am. Math. Soc. 106, 723-726 (1989)

12. Maluta, E: Uniformly normal structure and related coefficients. Pac. J. Math. 111, 357-369 (1984)

13. Lim, T-C, Xu, H-K: Uniformly Lipschitzian mappings in metric spaces with uniform normal structure. Nonlinear Anal., Theory Methods Appl. 25(11), 1231-1235 (1995)

14. Yao, J-C, Zeng, L-C: Fixed point theorem for asymptotically regular semigroups in metric spaces with uniform normal structure. J. Nonlinear Convex Anal. 8(1), 153-163 (2007)

15. Imdad, M, Soliman, AH: On uniformly generalized Lipschitzian mappings. Fixed Point Theory Appl. 2010, Article ID $692401(2010)$

16. Holmes, RD, Lau, AT-M: Nonexpansive actions of topological semigroups and fixed points. J. Lond. Math. Soc. 5 , 330-336 (1972)

17. Lau, AT-M, Takahashi, W: Invariant submeans and semigroups of nonexpansive mappings on Banach spaces with normal structure. J. Funct. Anal. 142(1), 79-88 (1996)

18. Lau, AT-M: Invariant means on almost periodic functions and fixed point properties. Rocky Mt. J. Math. 3(1), 69-75 (1973)

10.1186/1687-1812-2013-346

Cite this article as: Soliman: A tripled fixed point theorem for semigroups of Lipschitzian mappings on metric spaces with uniform normal structure. Fixed Point Theory and Applications 2013, 2013:346

\section{Submit your manuscript to a SpringerOpen ${ }^{\circ}$ journal and benefit from:}

- Convenient online submission

- Rigorous peer review

- Immediate publication on acceptance

Open access: articles freely available online

- High visibility within the field

- Retaining the copyright to your article 